\title{
Conhecimento e uso de tecnologias de verificação e controle de cores por editores de imagens digitais
}

\author{
Knowledge and use of color verification and control technologies \\ by digital image editors
}

Hugo Guilherme Pereira da Silva, Carla Patrícia de Araújo Pereira

cores digitais, controle de cores, editores de imagens

No campo do design, a tecnologia digital tornou-se amplamente difundida e incorporada à cultura de projeto, transformando as técnicas, materiais e processos próprios da atividade projetual, contexto em que o trabalho de manipulação de imagens se fortalece. Ao sair da materialidade dos suportes físicos e assumir formatos digitais, a edição de imagens trouxe necessidades específicas de manipulação das cores, o que exige constante atualização técnica para permitir explorar os recursos disponíveis, visando à qualidade dos produtos finais. A presente pesquisa investigou o nível de conhecimento de profissionais de edição de imagens a respeito das tecnologias de exibição e controle de cores. Foi realizado um estudo exploratório, de natureza qualitativa, com 64 profissionais de edição de imagens das diferentes regiões do país. Os resultados mostraram que a maioria desconhece a capacidade de exibição de cores do equipamento que utiliza em seus projetos e não realiza procedimentos de verificação e/ou checagem de cores.

digital colors, color control, image editors

In the field of design, digital technology has become widely disseminated and incorporated into the design culture, transforming the techniques, materials and processes proper to the project activity, a context in which the work of image manipulation is strengthened. By leaving the materiality of the physical media and assuming digital formats, the editing of images brought specific needs of color manipulation, which requires constant technical updating to allow to explore the available resources, aiming at the quality of the final products. This research investigated the level of knowledge of image editing professionals regarding color display and control technologies. An exploratory study of qualitative nature was carried out with 64 image editing professionals from different regions of the country. The results showed that most do not know the color display capacity of the equipment they use in their projects and do not perform color checking and/or checking procedures.

\section{Introdução}

A revolução digital teve um impacto irreversível nas formas de comunicação e nos métodos de trabalho, repercutindo em diversas áreas. No design, a tecnologia digital tornou-se amplamente difundida e incorporada à cultura de projeto, transformando substancialmente as técnicas, materiais e processos próprios da atividade projetual. Nos anos 1990, computadores com telas coloridas e softwares de edição estimulavam a reinvenção do design gráfico, proporcionando

Anais do $10^{\circ} \mathrm{CIDI}$ e $10^{\circ} \mathrm{CONGIC}$

Kelli C.A.S. Smythe, Rafael de Castro Andrade (orgs.)

Sociedade Brasileira de Design da Informação - SBDI

Curitiba | Brasil | 2021
Proceedings of the $10^{\text {th }} \mathrm{CIDI}$ and $10^{\text {th }}$ CONGIC

Kelli C.A.S. Smythe, Rafael de Castro Andrade (orgs.)

Sociedade Brasileira de Design da Informação - SBDI Curitiba | Brazil | 2021 
ferramentas para criação de tipografias, editoração e manipulação imagens coloridas (Meggs \& Purvis, 2009). Conforme explica Melo (2014, p. 612), a partir da revolução digital o designer "fortalece seu perfil de operador de imagens", particularmente fotografias, editadas através de recursos digitais. Nesse contexto, ao mesmo tempo em que tecnologia digital foi adotada "como ferramenta inovadora capaz de expandir as possibilidades e a própria natureza do processo do design" (Meggs \& Purvis, 2009, p. 629), as novas técnicas demandaram novas habilidades e conhecimentos.

Flusser (2007) ressalta que as superfícies digitais recebem hoje a importância que tinham os impressos em nosso passado recente. Os projetos não necessariamente geram um objeto material, de modo que todo o processo de criação e produção pode ocorrer no meio digital. Se, por um lado, as ferramentas digitais proporcionaram maior autonomia aos designers, elas trouxeram a necessidade de constante atualização técnica para permitir explorar os recursos disponíveis visando à qualidade dos produtos finais. Ao sair da materialidade dos suportes analógicos e assumir formatos digitais, o trabalho de edição de imagens trouxe necessidades específicas de manipulação e controle de cores.

Conforme Menezes e Pereira (2017, p. 322), as cores são elementos essenciais das imagens que participam da organização gráfica e da interpretação das mensagens visuais: "De um lado, atuam nos mecanismos da percepção para diferenciar, destacar e unificar outros elementos plásticos. De outro, por meio de associações mentais, as cores podem representar objetos concretos ou ideias abstratas, funcionando como signos". Nesse contexto, destaca-se a importância do controle de cores em projetos gráficos voltados ao meio digital, visando minimizar possíveis distorções entre as cores projetadas pelo designer e as cores visualizadas pelos usuários.

Diante do exposto, a presente pesquisa investigou o nível de conhecimento de profissionais de edição de imagens a respeito das tecnologias de exibição e controle de cores. Este artigo apresenta resultados parciais de pesquisa de mestrado desenvolvida na Universidade [Omitido para Revisão Cega] sobre a manipulação e gerenciamento de cores digitais na atividade de design, relatando um estudo exploratório em que foram entrevistados 64 profissionais de edição de imagens.

\section{Cores digitais}

Segundo Peddie (2013), a cor digital é uma reprodução de cor formada por informações binárias, sem a obrigatoriedade de uma referência analógica. Ela é gerada por um código ou comando que o sistema operacional do computador interpreta para ativar um percentual em cada canal de cor do monitor de referência: vermelho, verde e azul (RGB) (Peddie, 2013).

\section{Padrão CIE}

Conforme explica Leão (2005), com o crescimento da fabricação de telas por diversos fabricantes e marcas, fez-se necessária uma padronização internacional para se utilizar como 
referência para controle a exibição das cores em dispositivos. Esse padrão foi desenvolvido pela Comission Internationale de l'Eclairage (CIE), cujos desenvolvimentos mais importantes são: os sistemas padrão de colorimetria e observadores CIE 1931 e 1964; e os espaços de cores CIE 1931 XYZ, CIE 1979 L*a*b* e CIE 1976 L*u*v* (Arnkil, 2013).

Figura 1 - Diagrama CIE 1931

(Fonte: https://www.hisour.com/pt/ - Último acesso em 12/3/2020)

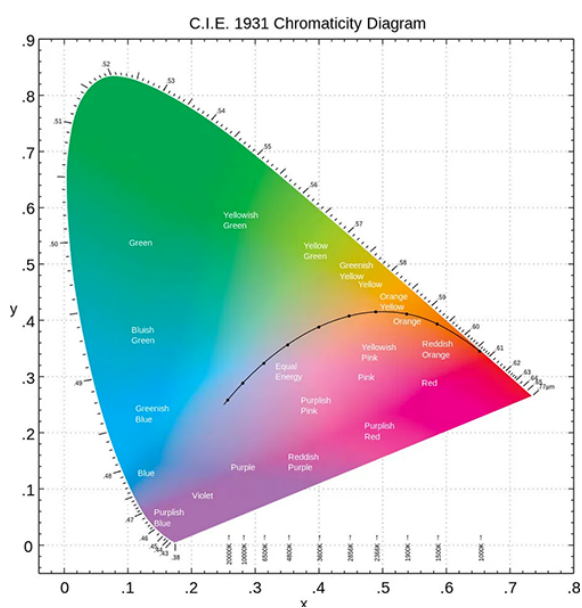

O diagrama CIE 1931 (Figura 1) foi desenvolvido para aferir com máxima precisão todas as cores que o humano normal consegue captar e perceber (Leão, 2005). Feisner e Reed (2014) esclarecem que o sistema CIE destaca-se de outros sistemas utilizados na indústria pela sua consistência e precisão na reprodução das cores.

\section{Espaços de cor}

Cada dispositivo é capaz de exibir ou captar um número limitado de cores. Esta fração quantificável é denominada espaço de cor. Monitores, sensores de câmeras digitais, scanners e outros dispositivos que intermediam ou reproduzem cores a partir de fontes digitais operam dentro de um determinado espaço. Os mais conhecidos são: CIELAB, Adobe RGB, sRGB e CMYK.

O espaço de cor CIELAB apoia-se no padrão do Internacional Color Consortium (ICC) ${ }^{1}$ definido pela CIE em 1976. Seu formato de mistura de cores baseia-se em três conjuntos de números que são identificados como coordenadas em cada um dos seus eixos tridimensionais (Sharma, 2018) (Figura 2). Este espaço busca uma aproximação com a visão humana, compreendendo uma maior gama de reprodução de cores. Trata-se de um espaço de cor independente, que exibe cores absolutas, os valores aplicados a ele são consistentes em qualquer dispositivo.

\footnotetext{
${ }^{1}$ International Color Consortium é uma comissão internacional criada para desenvolver e manter um sistema de gerenciamento de cores aberto e neutro, de forma equalizada em todos os sistemas operacionais e pacotes de software. Site oficial: http://www.color.org. Último acesso em 12/3/2020.
} 
Figura 2 - Diagrama tridimensional CIELAB

(Fonte: Michael Horvath. http://isometricland.net - Último acesso em 10/5/2020)

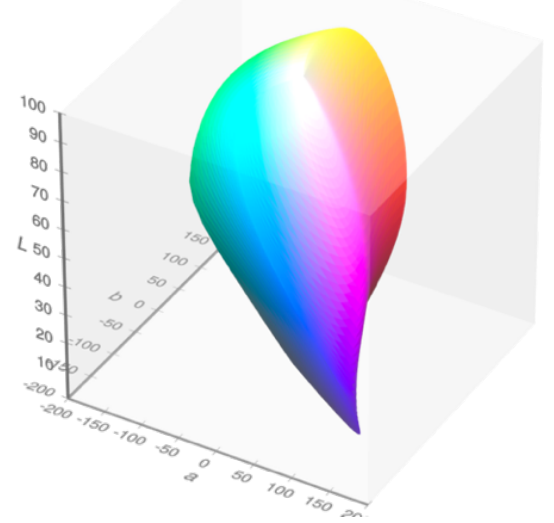

Criado pela Adobe Systems Inc. em 1998, o Adobe RGB foi projetado para compreender grande parte das cores disponíveis em impressoras coloridas CMYK, utilizando apenas cores primárias RGB em monitores e similares. Ele consegue exibir aproximadamente $50 \%$ das cores visíveis do CIELAB, variando de acordo com a capacidade do ecrã; sendo um dos espaços mais utilizados em câmeras digitais (Sharma, 2018). Os monitores mais sofisticados atualmente - indicados para necessidade de precisão na visualização das cores — conseguem exibir $99 \%$ ou a totalidade do espaço Adobe RGB.

Figura 3 - Comparativo Adobe RGB vs. sRGB

(Fonte: https://www.hisour.com/pt/ - Último acesso em 12/3/2020)

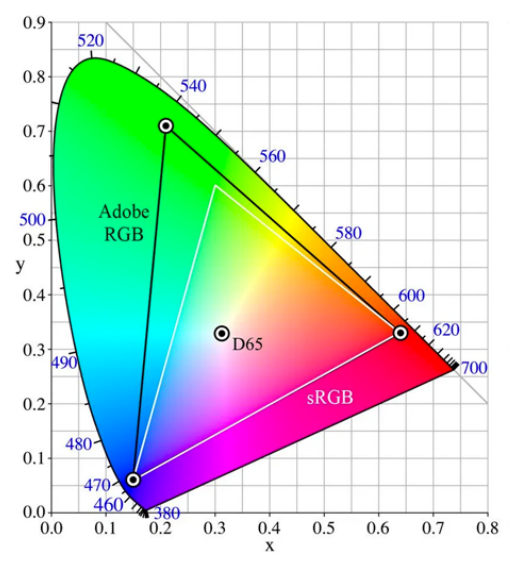

O sRGB (Standard Red Green Blue) foi criado pela Hewlett Packard e Microsoft em 1996 para ser utilizado em monitores, impressoras e padrões de exibição de cores em sites para internet. Sua principal característica de mistura de cores são os 255 níveis em cada um dos três canais de cores: vermelho, verde e azul. Apesar do alto número de cores disponíveis, é mais restrito que o Adobe RGB (Figura 3), predominantemente na gama dos matizes verdes e azuis. É um formato de segurança para trabalhar com arquivos de imagens em sites, já que os 
principais navegadores o reconhecem por padrão e exibem adequadamente, desde que o perfil seja incorporado ao arquivo (Ashe, 2014, p. 393).

O único espaço baseado no sistema cores subtrativas dentre os mencionados, o CMYK (cian, magenta, yellow, black), é o mais restrito. Amplamente utilizado nos processos de impressão, a quadricromia é limitada pela amplitude do espectro cromático do sistema. As cores visíveis pelo olho humano ou reproduzíveis em sistemas RGB não são alcançadas nesse espaço de cor. As misturas são realizadas por percentuais (de 0 a 100\%) em cada um dos quatro canais, formando uma cor resultante (Feisner \& Reed, 2014).

\section{Calibração das cores}

Os monitores de computadores evoluíram em paralelo com as ferramentas digitais, atingindo grandes formatos e altos níveis de resolução de tela e de qualidade de visualização. Alguns fabricantes desenvolvem monitores direcionados aos designers, com capacidades de exibição acima de 1 bilhão de cores. Nesse contexto, os calibradores de tela são recursos técnicos disponíveis para a verificação e checagem da cor, assim como os colorímetros, conforme descritos a seguir.

Os calibradores "são instrumentos que medem a transmitância ou refletância de uma superfície ou amostra em função do comprimento de onda" (Oliveira 2006, p. 92). A partir do reconhecimento das cores emitidas, eles são capazes de definir uma correção ideal para as perdas exibidas por monitores convencionais. Para tanto, o equipamento envia um ou mais comandos de correção para o sistema operacional em forma de parâmetros, os perfis ICC (Arnkil, 2013).

De acordo com Ang (2010), o perfil ICC é um arquivo de extensão *.icc ou *.icm que descreve a melhor maneira que um periférico deve reproduzir as cores, seja num monitor, projetor ou impressora. Ao final de cada calibragem, um novo arquivo é gerado pelo software do calibrador e adicionado ao sistema operacional para utilização. Os principais fabricantes já possuem perfis ICC genéricos ou pré-programados.

Feisner e Reed (2014, p. 58, tradução pelo autor) explicam que "o colorímetro é um instrumento que permite a medição da luz (RGB) e sua intensidade para combinar a cor como é percebida pelo olho humano. O colorímetro compara a cor medida com as cores padrão dentro da gama CIE", relacionando a precisão do dispositivo com os padrões internacionais de verificação cromática. Estes instrumentos utilizam três filtros para simular o comportamento da visão humana e medem a quantidade de luz que foi refletida pela amostra. Assim como os monitores, existem colorímetros específicos para calibragem de uso profissional (Feisner \& Reed, 2014).

Pelo exposto, considera-se que, com a elevada capacidade de exibição de cores pelos monitores atuais, o surgimento dos padrões estabelecidos pelos CIE e ICC, e os recursos técnicos disponíveis para mensuração das cores e calibragem de telas, erros de reprodução das cores podem ser reduzidos significativamente. Contudo, a maior precisão da exibição de 
cores e a consequente melhoria de qualidade do projeto dependem do conhecimento e uso de tais recursos pelos designers.

\section{Métodos e técnicas}

Foi realizado um estudo exploratório, de natureza qualitativa, com profissionais de edição de imagens de todas as regiões do país. Os dados foram coletados por meio de entrevistas semiestruturadas com 64 editores de imagens, homens e mulheres, entrevistados durante evento nacional ${ }^{2}$ direcionado a este público específico. A amostra foi não probabilística, por conveniência. A escolha dos respondentes se deu em função de sua disponibilidade e adesão espontânea a esta pesquisa. As respostas foram registradas em formulário previamente elaborado, preenchido pelo pesquisador, utilizando-se a plataforma Google Forms e smartphone conectado à internet. As entrevistas foram realizadas em espaços de convivência disponíveis durante o evento, com tempo médio de 2 minutos cada.

As questões iniciais levantaram o perfil dos entrevistados e as demais questões buscaram identificar o nível de conhecimento dos profissionais de edição de imagens a respeito das tecnologias de exibição e controle de cores. O tratamento estatístico foi realizado por meio de percentuais simples, seguindo-se de análise do tipo descritiva. Os dados obtidos nas entrevistas foram quantificados e organizados em tabelas e gráficos.

\section{Resultados e discussão}

A amostra de dados foi formada por editores de imagens que atuam no Brasil, sendo a maioria do sudeste $(59,38 \%)$, seguidos pelo nordeste $(17,19 \%)$, sul $(17,19 \%)$, e um menor percentual do centro-oeste $(4,69 \%)$ e norte $(1,56 \%)$. A maioria dos entrevistados $(53,3 \%)$ afirmou atuar como designer gráfico, havendo também fotógrafos $(10,9 \%)$, professores $(6,3 \%)$ e estudantes que atuam como freelancers (4,7\%). $24,8 \%$ dos entrevistados que não corresponderam às opções anteriores foram classificados na categoria "outros". Pouco mais de um terço $(34,4 \%)$ dos respondentes atua profissionalmente há mais de oito anos, $32,8 \%$ de três a oito anos, e $32,9 \%$ atuam há menos de três anos.

Sobre os tipos de arquivos gerados em seus projetos, a maioria $(62,5 \%)$ informou trabalhar tanto com arquivos para mídias digitais quanto com arquivos destinados à impressão. Os que trabalham exclusivamente com arquivos digitais somaram $37,5 \%$. Nenhum dos respondentes afirmou trabalhar exclusivamente com impressos. Estes dados mostram que a totalidade da amostra faz uso de meios digitais e que todos utilizam monitores para execução plena das atividades profissionais, inclusive as que envolvem cores.

Quanto à execução de procedimentos de checagem das cores entre o início do projeto e a finalização dos arquivos criados/editados, a maioria $(53,2 \%)$ informou não realizar qualquer

\footnotetext{
${ }^{2} 16^{\circ}$ Photoshop Conference, realizado de 27 a 29 de maio de 2019, em Campinas, São Paulo, Brasil.
} 
procedimento dessa natureza (Figura 4). Uma parcela menor $(23,4 \%)$ não sabia do que se tratava ou nunca ouviu falar sobre essa possibilidade. Outra parcela idêntica à anterior $(23,4 \%)$ respondeu que faz algum tipo de checagem. Para este último grupo, foi perguntado sobre o método utilizado para verificação das cores.

Figura 4 - Checagem e/ou validação das cores pela amostra (Fonte: Desenvolvido pelo autor)

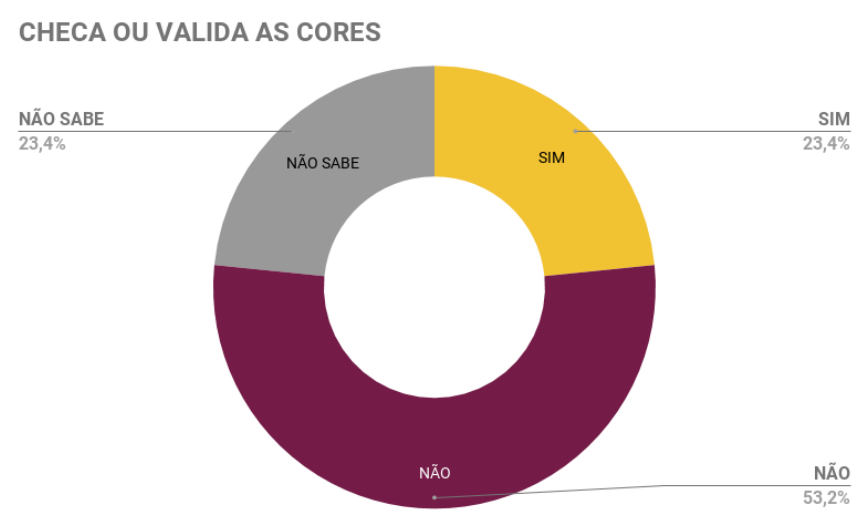

Dentre os respondentes que realizam verificação de cores, grande parte $(40 \%)$ afirmou fazer uso de pré-impressão ao desenvolver arquivos para mídia tradicional. Uma parcela equivalente (40\%) utiliza perfis de cores nos monitores. Apenas $20 \%$ dos que realizam a checagem de cores (Figura 5), fazem uso recomendado dos calibradores físicos em seus monitores, o que equivale a apenas $4,7 \%$ do total de entrevistados. É um percentual muito baixo, considerando que a totalidade da amostra trabalha com arquivos digitais e que $67,2 \%$ trabalham com edição de imagens há mais de três anos.

Figura 5 - Formas de verificação de cores de parte da amostra (Fonte: Desenvolvido pelo autor)

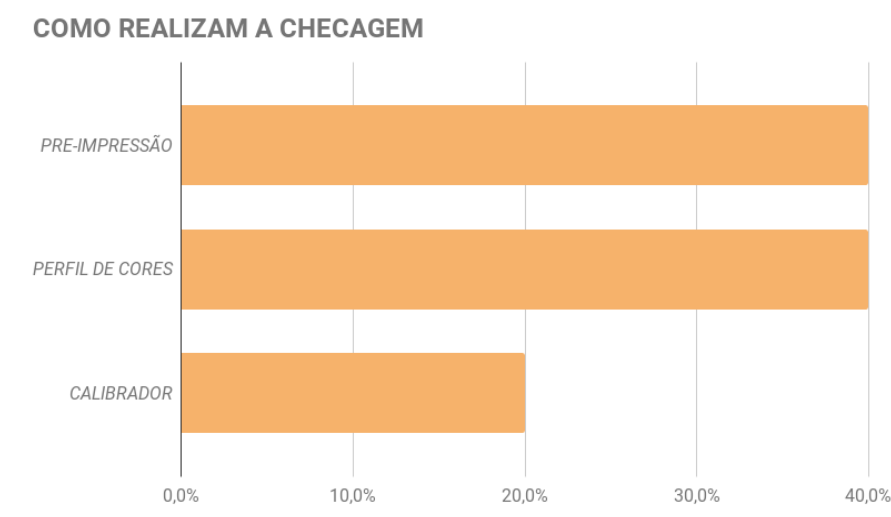

Ao serem questionados sobre a capacidade de exibição cores dos monitores que utilizam em seus projetos, a expressiva maioria (85,9\%) afirmou desconhecer (Figura 6). Em algumas 
respostas espontâneas foram detectadas dúvidas e surpresas sobre a existência dessas informações. Apenas nove entrevistados $(14,1 \%)$ responderam alguma alternativa válida.

Figura 6 - Respostas sobre a capacidade de exibição de cores dos monitores da amostra (Fonte: Desenvolvido pelo autor)

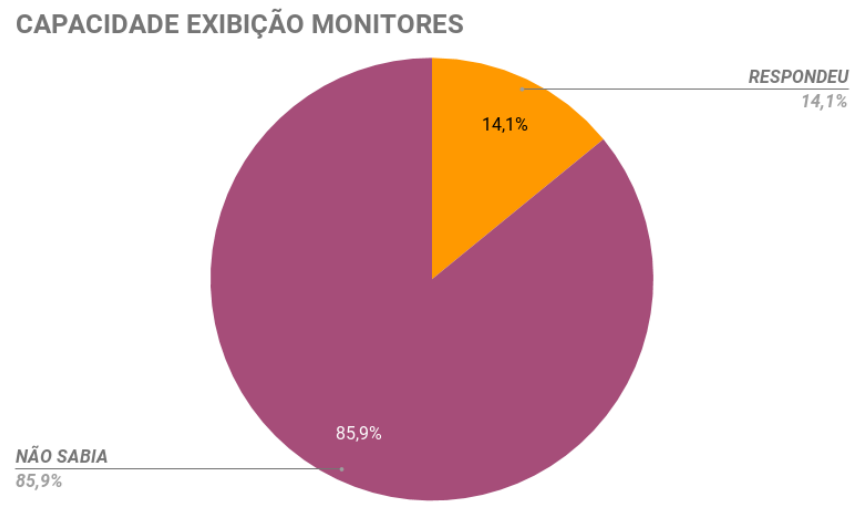

Apesar desse desconhecimento, a maior parte dos entrevistados $(60,9 \%)$ informou estar satisfeito com o equipamento, atribuindo nota 7,0 ou superior ao monitor que utiliza, considerando uma escala de zero a dez.

\section{Considerações finais}

A produção de imagens digitais e interação entre diferentes suportes requer conhecimento técnico visando à qualidade e produtividade. A necessidade de reinterpretar os matizes $\mathrm{e}$ tonalidades nos formatos digitais é tarefa diária para grande parte dos editores, designers e profissionais que trabalham com edição de imagens.

Discrepâncias entre as cores exibidas nos monitores em que os projetos são realizados e aquelas visualizadas outros suportes digitais ou mídias impressas podem ser minimizadas. Há notadamente um avanço tecnológico dos ecrãs e monitores, com capacidade crescente para exibição de cores, assim como tecnologia disponível para verificação e calibragem das cores. Entretanto, tais avanços têm passado ao largo do cotidiano de parte dos designers, apesar de trabalharem majoritariamente com imagens digitais.

Os dados mostraram um desconhecimento da maioria dos profissionais de edição de imagens entrevistados acerca dos recursos para que as cores projetadas no meio digital sejam reproduzidas corretamente, embora a amostra contemple, em sua maioria, profissionais com mais de três anos de experiência. Embora os resultados deste estudo não permitam generalizações, considerando sua amostragem não probabilística, deve-se destacar que as entrevistas foram realizadas com participantes de evento nacional de divulgação tecnológica, direcionados a indivíduos que buscam atualizações e avanços sobre recursos de sua área de atuação. 
Uma hipótese levantada a partir da análise dos dados sugere que esse grau de desconhecimento pode ter sua origem nas grades curriculares dos cursos que formam profissionais para criação em ambientes digitais, como design, mídias digitais, publicidade, e áreas correlatas. Desse modo, recomenda-se a continuidade da presente pesquisa, para a qual formulou-se a seguinte questão: os conteúdos abordados sobre cores nos cursos de formação superior em design no Brasil estão alinhados às atuais necessidades de uso das ferramentas digitais?

\section{Referências}

Ang, T. (2010). Fotografia digital masterclass. Rio de Janeiro: Alta Books. 360 p.

Arnkil, H. (2013). Colours in the visual world. Helsinki: Aalto Arts Books. 294 p.

Ashe, T. P. (2014). Color management \& quality output: working with color from camera to display to print. Burlington: Focal Press.

Feisner, E. A., \& Reed, R. (2014). Color studies. New York: Bloomsbury. 279 p.

Flusser, V. (2007). O mundo codificado: por uma filosofia do design e da comunicação. São Paulo: Cosac Naify. 224 p.

Leão, A. C. (2005). Gerenciamento de cores para imagens digitais. Dissertação (Mestrado em Artes Visuais) - Escola de Belas Artes, UFMG, Belo Horizonte. 135 p.

Meggs, P. B., \& Purvis, A. W. (2009). História do design gráfico. São Paulo: Cosac Naify. 720 p.

Melo, C. H. (2014). 1990-1999. In: MELO, Chico H. de; COIMBRA, Elaine R. (orgs.). Linha do tempo do design gráfico no Brasil. São Paulo: Cosac Naify. p. 612-696.

Menezes, H., \& Pereira, C. (2017). Funções da cor na infografia: uma proposta de categorização aplicada à análise de infográficos jornalísticos. Infodesign, v. 14. p. 321-339.

Oliveira, D. F. (2006). Confiabilidade metrológica e validação de procedimentos espectroradiométricos para medição de fontes luminosas. Dissertação (Mestrado - Área de Concentração: Metrologia para Qualidade e Inovação) - PUC-Rio, Rio de Janeiro. 192 p.

Peddie, J. (2013). The history of visual magic in computers: how beautiful images are made in cad, 3d, vr and ar. New York: Springer. 448 p.

Sharma, A. (2018). Understanding color management. 2. ed. New Jersey: Wiley, 2018. 344 p.

\section{Sobre os autores}

Hugo Guilherme Pereira da Silva, UFCG, Brasil <hugoguilherme@gmail.com>

Carla Patricia de Araújo Pereira, Dr. ${ }^{a}$, UFCG, Brasil <carlapereira.ufcg@gmail.com> 